\section{RISK FACTORS ASSOCIATED WITH ANXIETY AND DEPRESSION IN PROFESSIONAL CRICKETERS}

${ }^{1,2}$ Sharief Hendricks, ${ }^{1}$ Nur Amino, ${ }^{3}$ Ruan Schlebusch, ${ }^{3}$ JP Van Wyk, ${ }^{4}$ Stephen Mellalieu, 1,5,6Vincent Gouttebarge. 'Division of Exercise Science and Sports Medicine, Department of Human Biology, University of Cape Town, Cape Town, South Africa, Cape Town, South Africa; ${ }^{2}$ Institute for Sport, Physical Activity and Leisure, Leeds Beckett University, Leeds, UK, Leeds, UK; ${ }^{3}$ South African Cricketers' Association, Cape Town, South Africa, Cape Town, South Africa; ${ }^{4}$ Cardiff School of Sport and Heath Sciences, Cardiff Metropolitan University, Cardiff, UK, Cardiff, UK; ${ }^{5}$ Amsterdam UMC, Univ of Amsterdam, Department of Orthopaedic Surgery, Amsterdam Movement Sciences, Meibergdreef 9, Amsterdam, Netherlands, Amsterdam, Netherlands; ${ }^{6}$ Amsterdam Collaboration on Health and Safety in Sports (ACHSS), AMCNUmc IOC Research Center, Amsterdam, Netherlands, Amsterdam, Netherlands

\subsection{6/bjsports-2021-IOC.253}

Background In elite sport, mental health has become a topic of concern as athletes frequently appear to experience symptoms of anxiety/depression. Cricket is particularly demanding, given the globalisation and different formats of the game. To reduce anxiety/depression in professional cricketers, potential risk factors need to be identified.

Objectives Firstly, to determine the prevalence of anxiety/ depression in South African professional cricketers. Secondly, determine whether factors such as education, family life, or career-related factors are associated with anxiety/ depression.

Design A cross-sectional survey design using the General Health Questionnaire - a robust and reliable self-report measure for risk of anxiety/depression.

Participants All Professional South African Cricketers $(n=177)$. Assessment of Risk Factors Players' career (e.g. main role in the team, level of cricket), family (e.g. marital status, whether they had children) and education (e.g. highest level of education, whether they were currently studying)

Main Outcome Measurements General Health Questionnaire 12 (GHQ) Score (ranging from 0-12). Anxiety/Depression prevalence (based on GHQ). Relative Risk Ratios (RR) for anxiety/depression based playing career, family and education.

Results The prevalence of anxiety/depression was 58\%. The mean GHQ score for the sample was 3.6 (95\% CI: 3.2-4.0). The likelihood of developing anxiety/depression increased when players were playing a higher level (RR: 7.3; 95\% CI: $2.0-26.3 ; \mathrm{p}<0.01)$, contracted for more than 2 years (RR: $5.0 ; 95 \% \mathrm{CI}: 1.2-21.3 ; \mathrm{p}<0.05)$ or if they played their last offseason overseas (RR: 3.5; 95\% CI: 1.3-9.6; p < 0.05). The likelihood of developing anxiety/depression decreased when players made productive use of their spare time in the offseason (RR: 0.3; 95\% CI: 0.1-0.9; p < 0.05) and were contracted for 2 years (RR: 0.3 ; 95\% CI: $0.1-1.0$; $\mathrm{p}<0.05$ ). Conclusions The prevalence of symptoms of anxiety/depression in this cohort was higher than previously reported for elite athletes. Potential risk factors have been identified that can be used to design and develop strategies to reduce anxiety/depression in professional cricketers.

\section{ABSTRACT WITHDRAWN}

\section{EPIDEMIOLOGY OF HOSPITAL TREATED CRICKET- RELATED HAND INJURIES OVER A 5-YEAR PERIOD IN VICTORIA, AUSTRALIA}

${ }^{1}$ Karishma Shah, ${ }^{1,2,3,4}$ Nirmala Perera. ${ }^{1}$ Nuffield Department of Orthopaedics, Rheumatology and Musculoskeletal Sciences, University of Oxford, Oxford, UK; ${ }^{2}$ Centre for Sport, Exercise and Osteoarthritis Research Versus Arthritis, Oxford, UK; ${ }^{3}$ Division of Physiotherapy, Department of Medical and Health Sciences, Linköping University, Linköping, Sweden; ${ }^{4}$ School of Allied Health, College of Science, Health and Engineering, Latrobe University, Melbourne, Australia

\subsection{6/bjsports-2021-IOC.254}

Background Cricket is one of the most popular sports worldwide. However, the risk of hand injuries is high. For example, batters can be struck, wicketkeepers must catch the fast-moving ball, and fielders might dive to stop the ball. In retired elite cricketers, previous search shows 36\% report severe hand injury, and this is associated with pain (20\%) and osteoarthritis $(2 \%)$. Hand osteoarthritis is known to decrease quality of life, and increase morbidity. The majority of cricketers are recreational players. However, the epidemiological data on cricket-related hand injuries are under reported in this population. This data are needed to inform development of evidence-based injury prevention programmes.

Objective Present the first comprehensive epidemiological profile of hospital treated cricket-related hand injuries from 2013-14 to 2017-18 in Victoria, Australia.

Design Retrospective analysis of routinely collected hospital presentation data (detailed case-series).

Setting De-identified hospital-treatment data from two hospital databases: Victorian Emergency Minimum Dataset (ED-presentations) and Victorian Admitted Episodes Dataset (hospital-admissions), were obtained from the Victorian Injury Surveillance Unit. Participants Male and female cricketers.

Main Outcome Measurements Incidence and description of ED-presentations and hospital-admissions.

Results 2,415 cases (1,960 ED-presentations) were treated in Victorian hospitals. $96 \%$ of cases were male. All $(n=455)$ hospital-admissions were for one day. Most frequent ED-presentations were in the -15-19 year age group, whilst the 25-29 year ago group reported most frequent hospital-admissions. Fractures (48\% ED-presentations versus 73\% hospital-admissions) were the commonest injury type. Thumb injuries (17\%) were most frequently treated in EDs. The most common injury mechanism was being hit/stuck/crushed (84\% ED-presentations versus 79\% hospital admissions).

Conclusions Cricket-related hand injuries are more frequent in younger players. Fractures are the most common type of injury, accounting for half of all ED-presentations. Fractures are also the most severe injury, accounting for 3 out of 4 hospital admissions. Hand injury prevention strategies need to be prioritised in recreational cricketers.

\section{IMPACT OF SPECIFIC PREVENTION TRAINING MEASURED BY THE STAR EXCURSION BALANCE TEST MODIFIED (SEBTM) IN YOUNG FEMALE SOCCER: A CLUSTER RANDOMISED CONTROLLED TRIAL}

${ }^{1}$ Hugo Del Rabal, ${ }^{3}$ Brice Picot, ${ }^{2}$ Alexandre Rambaud. 'Société Française des MasseurKinésithérapeutes du Sport, Tain I'Hermitage, France; ${ }^{2}$ Société Française des MasseurKinésithérapeutes du Sport, La Talaudière, France; '35ociété Française des MasseurKinésithérapeutes du Sport, Chambéry, France 
Background Use of SEBTm can identify athletes with risk of injury and help to set up prevention strategies.

Objective To identify subjects at risk of lower limb injuries (LLI) with SEBTm after a specific prevention training.

Design Cluster randomised controlled trial.

Setting French elite soccer youth academy.

Patients (or Participants) 36 healthy female athletes (aged 1519 years old) were included and assigned to the 'Control Groupe' (CrtGrp) or 'Prevention Training Group' (PrevGrp) by a cluster randomised controlled trial.

Interventions (or Assessment of Risk Factors) Both groups performed normal 5 weeks pre-season training, but in the intervention' we implemented a specific prevention protocol based on international recommendations.

Main Outcome Measurements Composite score (CS) of SEBTm were measured in both group at baseline (T0), after 5 weeks (post-protocol) and 3 months after the end of the protocol. Furthermore a 10 month follow up questionnaire post protocol was used to asses LLI in the two groups.

Results After 5 weeks and exclusion criteria, 28 players completed the all study (16 in the control group and 12 in the intervention group). After the protocol period, CS increased significantly in the PrevGrp $(p=0,03)$. In the CrtGrp, no difference was seen between the 2 testing sessions $(p=0,68)$. Based on injury risk factors, 19 subjects were identified as 'at risk' at T0. Of these, 4 players had at-least 1 LLI after 10 months $(R R=0,67)$. Moreover, 2 subjects who were not identified 'at risk' sustained a LLI during during the 10 months period. After 5 weeks, 2 of the 12 subjects in the PrevGrp exceeded the SC cut-off, compare to 0 on 16 players in the CrtGrp.

Conclusions This study suggests that the prevention training program seems to improve the SC at SEBT. This could leed to a diminished risk to sustain an LLI. These results needed to be confirmed with larger sample size studies.

\section{USING INFOGRAPHICS AND VIDEO TO PREVENT INJURIES AND ILLNESSES IN ATHLETICS}

1,2,3,4,5 Pascal Edouard, ${ }^{5}$ Pedro Branco, ${ }^{6,7}$ Danny Glover, ${ }^{6} J e n n i f e r$ Duncan, ${ }^{8}$ Andy Richardson, ${ }^{5,9,10}$ Marianna Kiss, ${ }^{3,12}$ Frederic Depiesse, ${ }^{6,11}$ Andrew Murray. ${ }^{1}$ Interuniversity Laboratory of Human Movement Science (LIBM EA 7424), University of Lyon, University Jean Monnet, Saint-Etienne, France; ${ }^{2}$ Department of Clinical and Exercise Physiology, Sports Medicine Unit, University Hospital of Saint-Etienne, Faculty of Medicine, Saint-Etienne, France; ${ }^{3}$ Medical Commission, French Athletics Federation (FFA), Paris, France; ${ }^{4}$ Swiss Olympic Medical Centre, Centre de médecine du sport, Division de médecine physique et réadaptation, Centre Hospitalier Universitaire Vaudois, Lausanne, Switzerland; ${ }^{5}$ European Athletics Medical and Anti-Doping Commission, European Athletics Association (EAA), Lausanne, Switzerland; ${ }^{6}$ Knowledge Translation Team, Sport and Exercise, University of Edinburgh, Edinburg, UK; ${ }^{7}$ Health Education Yorkshire and the Humber, Leeds, UK; ${ }^{8}$ Institute of Cellular Medicine, Newcastle University, Newcastle Upon Tyne, UK; ${ }^{9}$ Hungarian Athletics Federation (Magyar Atlétikai Szovetség), Budapest, Hungary; ${ }^{10}$ National Institute for Sport Medicine, Budapest, Hungary; ${ }^{11}$ Human Performance Science Research Group, University of Edinburgh, Edinburg, UK; ${ }^{12}$ Department of Physical Medicine and Rehabilitation, University Hospital of Martinique, Le Lamentin, Martinique, France

\section{$10.1136 /$ bjsports-2021-IOC.256}

Background Injuries and illnesses represent a health problem affecting athletics athletes' training and performance. Recently, research on injury and illness epidemiology and risk factors have provided some insights to help implement prevention strategies. The next step is presenting the science, in an engaging form that can assist medical teams, coaches and athletes understand and implement these strategies.
Objectives To produce resources in order to disseminate knowledge on injuries and illnesses epidemiology and risk factors, and highlight potential prevention strategies.

Participants Researchers and practitioners working in athletics injury and illness prevention collaborated with athletes.

Main Outcome Measurements Resources/communication assets, for example infographics, animations, video, were produced and shared with medical teams, athletes and coaches.

Results Infographics, animation and engaging knowledge translation tools/educational materials on illness epidemiology during international athletics championships were produced. These took the scientific knowledge, made it specific for the context of athletics, and provided easily digestible, visually rich information. These were shared with athletes at events such as the European Athletics Indoor Championships.

Conclusions We developed resources to be shared to all stakeholders working with athletics athletes, providing tips and practical recommendations on injury and illness prevention. Having share these to athletes and their teams, the next step is to evaluate the documents and tools for and by athletes and involved actors, and the effectiveness of these interventions. These strategies may be relevant to provide athletes with engaging information on preventing injury and illness in other sports.

\section{FIELD HOCKEY INJURIES AND PERSONAL PROTECTIVE EQUIPMENT: A STATUS SURVEY OF GERMAN NATIONAL TEAMS}

Sabrina Gorks, Thomas Henke, Petra Platen. Ruhr-University Bochum, Dep. of Sports Medicine, Bochum, Germany

\subsection{6/bjsports-2021-IOC.257}

Background In order to prevent sports injuries, it is necessary to know the number of injuries and injury mechanisms of sports-related injuries. In addition, acceptance and use of personal protective equipment (PPE) should be examined. The results give a better overview in German elite hockey.

Objective Data on injury frequencies and injury patterns in field hockey as well as on the wearing behavior of PPE in training and competition.

Design Questionnaire-based retrospective total survey. Players were instructed to fill out one general and, in case of at least one time loss injury (at least 1 training or competition missed) during a 3-year-period (February 2016 - April 2019), one additional questionnaire per injury.

Setting National elite hockey level. Participants were member of the German national teams including A-, U 21- and U 18men and women teams (age range 16-32y).

Participants 127 players took part in the survey.

Main Outcome Measurements Exposure, injured body parts, incidence, time loss, wearing of PPE

Results 89 players sustained 114 injuries. Incidence: Competition: Men: 5.79 injuries per 1000h; Women: 7.99 injuries per 1000h. Training: Men: 0.51 injuries per 1000h; Women: 0.31 injuries per 1000h. Body parts: Men: 77.6\% lower extremities, 8.95\% upper extremities. Women: 75\% lower extremities, $16.7 \%$ upper extremities. Severity defined by Fuller et al. (2006): Men: 53.3\% severe, 30\% moderate, $10 \%$ mild and $6.67 \%$ minimal. Women: $47.8 \%$ severe, 37\% moderate, $13 \%$ mild and $2.17 \%$ minimal. PPE: Fielders: Men: leg protection 96.6\%, mouthguard $88.1 \%$ and gloves $66.1 \%$. Women: leg protection 98\%, mouthguard $94 \%$ and gloves 94\%. Goalkeeper: carry more PPE in addition to mandatory PPE. 